\title{
PRODUTIVIDADE E ABSORÇÃO DE FÓSFORO POR PLANTAS DE MILHO EM FUNÇÃO DE DOSES E MODOS DE APLICAÇÃO DE ADUBO FOSFATADO EM SOLO DE TABULEIRO COSTEIRO(1)
}

\author{
A. C. BARRETO(2) \& M. F. FERNANDES ${ }^{(2)}$
}

\begin{abstract}
RESUMO
O objetivo deste estudo foi avaliar os efeitos de doses e modos de aplicação de $\mathbf{P}$ ao solo sobre a absorção e a eficiência de utilização do $\mathbf{P}$ foliar $\mathbf{e}$ a produtividade de milho (Zea mays) cultivado em um Argissolo Amarelo, característico dos tabuleiros costeiros. Avaliaram-se as doses de 0, 45, 90, 135 e $180 \mathrm{~kg} \mathrm{ha}^{-1}$ de $\mathrm{P}_{2} \mathrm{O}_{5}$, distribuídas no sulco de plantio ou a lanço. $\mathrm{O}$ experimento foi realizado na estação chuvosa, de junho a setembro de 1998, no Campo Experimental de Umbaúba (E mbrapa Tabuleiros Costeiros), no município de Umbaúba (SE ). A adubação fosfatada a lanço resultou em maiores teores de P foliar das plantas de milho, comparativamente à adubação em sulco. E m virtude desta maior capacidade de absor ção de $\mathrm{P}$, as plantas adubadas a lanço produzi ram maior quantidade de grãos e apresentaram menor requerimento de adubos fosfatados para alcançar o máximo de produtividade. A eficiência de utilização do $P$ foliar para produção de grãos de milho foi influenciada apenas pela dose de $P$, não sendo alterada pelo modo de adubação fosfatada. A partir da dose de 114,3 kg ha-1 de $\mathrm{P}_{2} \mathrm{O}_{5}$, os incrementos no teor de $\mathrm{P}$ foliar não resultaram em aumento da eficiência de utilização do $P$ foliar para a produtividade de grãos. A avaliação da produtividade e a distribuição de raízes, realizadas apenas nos tratamentos com dose de $90 \mathrm{~kg} \mathrm{ha}^{-1}$ de $\mathrm{P}_{2} \mathrm{O}_{5}$, revelaram que a adubação a lanço resultou em maior biomassa radicular e uma tendência em melhor distribuição destas raízes.
\end{abstract}

Termos de indexação: Zea mays, adubação fosfatada, métodos de adubação, eficiência de utilização de $P$, distribuição radicular.

(1) Trabalho apresentado no XXVII Congresso Brasileiro de Ciência do Solo, realizado em Brasília (DF), de 11 a 16 de julho de 1999. Recebido para publicação em março de 2000 e aprovado em setembro de 2001.

(2) Pesquisador da E mbrapa Tabuleiros Costeiros. Caixa Postal 44, CEP 49001-970Aracaju (SE). E-mails: barreto@cpatc.embrapa.br; marcelo@cpatc.embrapa.br 


\title{
SUMMARY: YIELD AND PHOSPHORUS UPTAKE BY CORN PLANTS AS AFFECTED BY PHOSPHORUS FERTILIZER RATES AND PLACEMENT IN A COASTAL TABLELAND SOIL
}

\begin{abstract}
The objective of this study was to evaluate the effects of $P$ rates and placement on $P$ uptake, leaf P use efficiency (PUE), and grain yield of corn (Zea mays) grown in a Yellow Argissol soil, typical of the coastal tableands. Rates of $0,45,90,135$ and $180 \mathrm{~kg} \mathrm{ha}^{-1}$ of $\mathrm{P}_{2} \mathrm{O}_{5}$ were applied in either the rows or broadcast. The study was carried out during the rainy season, from J une to September of 1998, at Umbaúba Experimental Station, at U mbaúba (SE), Brazil. Broadcast $P$ fertilization resulted in higher leaf $P$ concentration, compared to therow $\mathrm{P}$ fertilization. In responseto this higher $\mathrm{P}$ uptake, broadcast-fertilized plants yiedded more grains and presented a lesser requirement of $P$ fertilizer to reach the highest productivity. PUE was affected by theP rates, but not by $P$ placement. I ncreases of leaf $\mathrm{P}$ concentration in response to rates higher than $114.3 \mathrm{~kg} \mathrm{ha}^{-1}$ of $\mathrm{P}_{2} \mathrm{O}_{5}$ did not result in increases in PUE. Root evaluation, performed only at therate of $90 \mathrm{~kg} \mathrm{ha}^{-1}$ of $\mathrm{P}_{2} \mathrm{O}_{5}$, showed that broadcast $P$ fertilization resulted in higher root dry weight and in a better root distribution.
\end{abstract}

Index terms: Zea mays, phosphorus fertilization, fertilization methods, phosphorus use efficiency, root distribution.

\section{INTRODUÇÃO}

A localização do adubo fosfatado em relação às raízes das plantas é fator determinante para a absorção deP, crescimento e produtividade demuitas culturas (Borkert \& Barber, 1985; Anghinoni, 1992; Silva et al., 1993).

A mistura doadubo fosfatado com mai ores frações de sol o tem, de modo geral, resultado em aumento da utilização dos fertilizantes fosfatados por plantas de milho (Novais et al., 1985; Anghinoni, 1992; Klepker \& Anghinoni, 1996). Este aumento é atribuído ao contato entre a maior quantidade de raízes e ofertilizantefosfatado (Anghinoni \& Barber, 1980; Klepker \& Anghinoni, 1993), o que reduz as distâncias percorridas pelo $\mathrm{P}$ até às raízes. $\mathrm{Em}$ virtude da baixa mobilidade do P no solo, este contato raízes- $P$ é determinante para a eficiência de utilização dos adubos fosfatados (Stryker et al., 1974).

No entanto, o volume de sol o a ser adubado para otimizar a utilização de fertilizantes fosfatados depende da dosagem deste fertilizante (Anghinoni \& Barber, 1980; Anghinoni, 1992). De acordo com esses autores, dosagens reduzidas devem ser aplicadas localizadamente, já que a diluição excessiva do $\mathrm{P}$ com o solo pode aumentar sua adsorção ea sua passagem para as formas não-lábeis. O efeito positivo desta localização do P em um volume restrito de sol o será mai or quanto maior for o fator capacidade de P (FCP) do solo (Anghinoni \& Barber, 1980; Novais \& Smyth, 1999).

Considerando que os sol os de tabulei ros costei ros apresentam, de modo geral, baixos teores de óxidos de ferro e de alumínio em superfície, bem como reduzidos teores de argila, comparativamente a diversos sol os ácidos tropicais, acredita-se que a aplicação de P a lanço seja uma alternativa capaz deincrementar a eficiência de utilização dos adubos fosfatados, mesmo quando doses reduzidas forem usadas.

O objetivo deste trabalho foi avaliar o efeito de diferentes doses e modos de aplicação de adubos fosfatados sobre a produtividade, sobre a absorção de $P$ e sobre a eficiência de utilização do $P$ foliar por plantas de milho.

\section{MATERIAL E MÉTODOS}

O experimento foi realizado de junho a setembro de 1998, durante a estação chuvosa, em Campo Experimental da Embrapa - Tabuleiros Costeiros, localizado no município de Umbaúba (SE). A precipitação pluviométrica neste período foi de $912 \mathrm{~mm}$, enquanto a média da região, nos últimos cinco anos, foi de $665 \mathrm{~mm}$.

O solo da área utilizada foi classificado como Argissolo Amarelo, apresentando as seguintes características químicas na camada de $0-20 \mathrm{~cm}$ de profundidade: $\mathrm{pH}$ em água $(1: 2,5)=5,5$; matéria orgânica (J ackson, 1958) $=30 \mathrm{~g} \mathrm{dm}^{-3} ; \mathrm{P}=1 \mathrm{mg} \mathrm{dm}^{-3}$ e $\mathrm{K}=25 \mathrm{mg} \mathrm{dm}^{-3}$, extraídos pelo Mehlich-1; $\mathrm{Ca}=$ $0,8 \mathrm{cmol}_{\mathrm{c}} \mathrm{dm}^{-3}$ e $\mathrm{Mg}=0,2 \mathrm{cmol}_{\mathrm{c}} \mathrm{dm}^{-3}$, extraídos com $\mathrm{KCl} 1 \mathrm{~mol} \mathrm{~L}{ }^{-1} ; \mathrm{Al}=0,1 \mathrm{cmol}_{\mathrm{c}} \mathrm{dm}^{-3}$, extraído com KCl $1 \mathrm{~mol} \mathrm{~L}^{-1}$, e H $+\mathrm{Al}=3,0 \mathrm{cmol}_{\mathrm{c}} \mathrm{dm}^{-3}$, extraídos por acetato de cál cio 0,5 mol L-1, tamponado em $\mathrm{pH}$ 7,0 (Silva et al, 1999). Os resultados da análise 
granulométrica, pelo método do densímetro (EMBRAPA, 1997), foram os seguintes: areia muito grossa $=720,6 \mathrm{~g} \mathrm{~kg}^{-1}$; silte $=229,4 \mathrm{~g} \mathrm{~kg}^{-1}$ e argila $=$ $50,0 \mathrm{~g} \mathrm{~kg}^{-1}$. Deacordo com estes resultados, atribuiuse a este solo a classificação textural de franco arenoso.

Os tratamentos consistiram de um arranjo fatorial $5 \times 2$, avaliando-se cinco doses de $\mathrm{P}(0,45$, 90,135 e $180 \mathrm{~kg} \mathrm{ha}^{-1}$ de $\mathrm{P}_{2} \mathrm{O}_{5}$ ), adicionadas ao solo de duas formas distintas: no sulco de plantio ou a lanço. Estes tratamentos foram dispostos de acordo com um delineamento de blocos ao acaso, com três repetições. Cada parcela experimental abrangeu uma área total de 43,2 $\mathrm{m}^{2}$ (5,4 × 8 m), com seis linhas espaçadas de 0,9 m. A parcela útil foi composta pelas plantas das quatro linhas centrais da parcela total, descontado $1 \mathrm{~m}$ de cada extremidade destas linhas.

E m maio, 40 dias antes do plantio, a área recebeu 1 t ha-1 de calcário dolomítico com PRNT de $80 \%$, incorporado até, aproximadamente, $20 \mathrm{~cm}$ de profundidade. A necessidade de calagem foi determinada de acordo com o método da neutral ização do alumínio e el evação de cálcio e magnésio.

A adubação fosfatada foi feita imediatamente antes do plantio do milho com superfosfato triplo. Nos tratamentos com adição de $\mathrm{P}$ a Ianço, $\mathrm{O}$ fertilizante foi distribuído manualmente e incorporado até cerca de $20 \mathrm{~cm}$ de profundidade, utilizando duas gradagens. Nas demais parcelas, o superfosfato tripl o foi adicionado no fundo do sulco de plantio, a cerca de $10 \mathrm{~cm}$ de profundidade.

Independentemente da forma com que o $\mathrm{P}$ foi adicionado ao solo, as adubações de plantio para fornecimento de $\mathrm{N}$ eK também foram feitas no fundo do sulco.

$\mathrm{O} \mathrm{K}$ foi fornecido na forma de $\mathrm{KCl}$, em quantidade equivalente a $75 \mathrm{~kg} \mathrm{ha}^{-1}$ de $\mathrm{K}_{2} \mathrm{O}$, e o N, como sulfato de amônio, em quantidade equivalente $80 \mathrm{~kg} \mathrm{ha}^{-1}$. A aplicação desses fertilizantes foi feita em duas parcelas, sendo a primeira realizada no sulco de plantio, utilizando $1 / 3$ da dosagem total, ea segunda, em cobertura, 1 mês após o plantio, utilizando os 2/3 restantes.

Três sementes de milho (variedade Sertanejo) foram plantadas a cada espaço de $40 \mathrm{~cm}$ dentro da linha. Após 25 dias do plantio, procedeu-se ao desbaste do experimento, deixando-se duas plantas por cova, com vistas em obter uma população final aproximada de 55.500 plantas ha-1.

Os teores de $\mathrm{P}$ nas plantas de milho foram determinados de acordo com Malavol ta et al. (1989), utilizando o terço mediano das folhas +4 de 12 plantas por parcela, amostradas no início da emissão das inflorescências femininas, 62 dias após emergência.

Para avaliar o efeito das formas de adição do $P$ ao solo sobre a distribuição lateral das raízes de milho na camada arável, retiraram-se amostras de solo perpendicularmente à linha de plantio, a cada $10 \mathrm{~cm}$, até uma distância de $50 \mathrm{~cm}$ das plantas, de ambos os lados do sulco. A largura destas amostras foi de $25 \mathrm{~cm}$ e a profundidade de $20 \mathrm{~cm}$. Apenas as parcelas que receberam dose de $P$ equivalente a $90 \mathrm{~kg} \mathrm{ha}^{-1}$ de $\mathrm{P}_{2} \mathrm{O}_{5}$ foram amostradas. Para esta variável, os tratamentos consistiram de um arranjo fatorial $5 \times 2$, avaliando-se cinco distâncias em relaçãoà planta $(0-10,10-20,20-30,30-40$ e 40-50 cm) e os dois métodos de aplicação do $\mathrm{P}$ no solo.

A eficiência deutilização do $P$ (EUP) pelas plantas foi empregada para expressar a produtividade de grãos por planta em relação a uma unidade percentual de $P$ na matéria seca de folhas. O uso desta variável foi adaptado deSiddiqi \& Glass (1981), que empregaram a razão entre produtividade de folha e teor de nutriente foliar para determinar a eficiência de utilização.

\section{RESULTADOS E DISCUSSÃO}

Os teores de $\mathrm{P}$ foliar foram influenciados tanto pelas doses quanto pelo modo de adubação com $\mathrm{P}$, não tendo sido observado efeito significativo da interação dos dois fatores sobre esta variável (Quadro 1). Para ambos os modos de aplicação do P, observaram-se incrementos lineares dos teores fol iares deste nutriente em resposta às doses de $\mathrm{P}_{2} \mathrm{O}_{5}$ no solo (Figura 1). A pós aval iação da homogeneidade dos coeficientes de regressão (Gomez \& Gomez, 1976), observou-se que os coeficientes lineares das equacões de resposta das plantas às doses crescentes de $\mathrm{P}_{2} \mathrm{O}_{5}$ em cada um dos métodos de adicão de $\mathrm{P}$ ao solo diferiram pel o teste-t, a $5 \%$.

Por ter sido essa resposta mais acentuada para as plantas adubadas a lanço (Figura 1), concluiu-se que este modo de adubação fosfatada resultou em maior eficiência de absorção do $P$ pelas plantas de milho, comparativamente à adubação em sulco. O aumento da absorção de $\mathrm{P}$ em conseqüência da incorporação do adubo fosfatado com um volume maior de solotem sido relatado por diversos autores, que relacionam este efeito positivo ao maior comprimento de raízes em contato com o adubo fosfatado no sol o (Anghinoni \& Barber, 1980; K lepker \& Anghinoni, 1993). Este efeito tenderá a ser mais expressivo com o aumento das doses de fertilizantes utilizadas ou com a redução do poder de adsorção de P do solo (Anghinoni \& Barber, 1980).

Os resultados de teores foliares de $\mathrm{P}$ do presente estu do corroboram estas observações, já que, quanto menores as doses de $\mathrm{P}_{2} \mathrm{O}_{5}$ adicionadas ao solo, menor o benefício da adubação fosfatada a lanço, em rel ação à feita no sulco (Figura 1). No entanto, mesmo a adubação a lanço com doses reduzidas de $\mathrm{P}_{2} \mathrm{O}_{5}$ (50 kg ha-1), que resultaria em grande diluição do 
Quadro 1. Análise de variância da produtividade de grãos, do teor de P foliar e da eficiência de utilização do P (E UP) pelo mi lho considerando diferentes doses e forma de adição de P ao solo

\begin{tabular}{|c|c|c|c|c|}
\hline \multirow{2}{*}{ F.V. } & \multirow{2}{*}{ G.L. } & \multicolumn{3}{|c|}{ Quadrado médio } \\
\hline & & Produtividade de grão & Teor de $P$ foliar & EUP \\
\hline Bloco & 2 & $1.230 .083,84^{*}$ & $0,0009667 \mathrm{~ns}$ & $22.832,23^{n s}$ \\
\hline Forma de adição do $P(A)$ & 1 & $2.064 .007,20 *$ & $0,0048387^{* *}$ & $1.840,83^{\text {ns }}$ \\
\hline Dose de $P(D)$ & 4 & $13.325 .225,70 * *$ & $0,0131888 * *$ & $124.540,75^{* *}$ \\
\hline$A \times D$ & 4 & $196.650,99^{\text {ns }}$ & $0,0005743^{\text {ns }}$ & $1.547,58^{\text {ns }}$ \\
\hline Resíduo & 18 & $252.491,76$ & 0,0003773 & $4.939,97$ \\
\hline Total & 29 & & & \\
\hline C.V. (\%) & & 18,31 & 10,51 & 23,92 \\
\hline
\end{tabular}

**, * e ns: Significativos a 1 e 5\% e Não-significativo, respectivamente, pelo teste F.

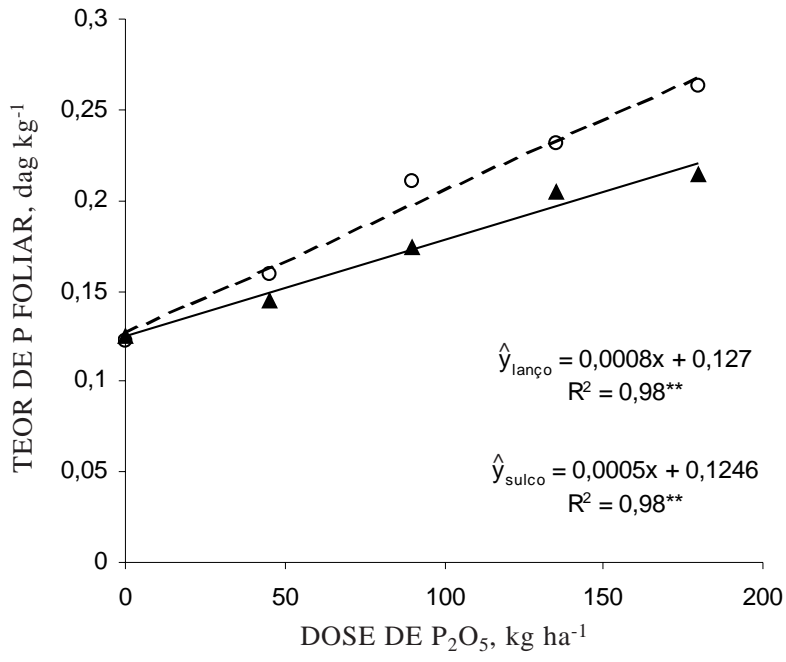

Figura 1. Teor de $P$ em folhas de milho em resposta a doses de $\mathrm{P}_{2} \mathrm{O}_{5}$ adicionadas a lanço (---) e no sulco de plantio (-) em um solo de tabuleiro costeiro.

nutriente no solo e, conseqüentemente, em menor disponibilidade do nutriente em solução, propiciou uma absorção superior de $\mathrm{P}$ pelas plantas, comparativamente à adubação no sulco de plantio. Este efeito provavelmente está relacionado com a baixa capacidade de adsorção de P do solo em estudo, o que permitiu que, mesmo sob esta condição de grande di luição do adubo fosfatado, as concentrações deP em solução ainda fossem suficientes para prover $\mathrm{P}$ de modo mais eficiente do que a adubação em sulco.

Todavia, pela tendência de redução do efeito positivo da adubação a lanço observada com a redução das doses de $\mathrm{P}_{2} \mathrm{O}_{5}$, esperava-se que, para este mesmo sol o, a aplicação em sulco de doses de $P$ inferiores a $50 \mathrm{~kg} \mathrm{ha}^{-1}$ de $\mathrm{P}_{2} \mathrm{O}_{5}$ produzisse uma eficiência deabsorção maior ou equival enteà obtida na adubação a lanço.
A produtividade degrãos apresentou, para ambos os modos de adubação, resposta quadrática às doses $\operatorname{de}_{2} \mathrm{O}_{5}$ nosolo (Figura 2). Plantas adubadas com P a Ianço al cançaram maiores produtividades, refletindo a maior capacidade de absorção de $\mathrm{P}$ apresentada por elas, comparativamente às adubadas com $P$ no sulco de plantio. Efeitos negativos da adubação localizada de $\mathrm{P}$ sobre a produtividade têm sido atribuídos, por alguns autores, à salinidade ou à toxidez, resultantes das altas concentrações dos adubos fosfatados, o que afetaria a germinação das sementes (Miller et al., 1971) e o crescimento das raízes (Peryea, 1990).

Segundo as equações de regressão estimadas, a produtividade máxima de grãos obtida seria de $4.260 \mathrm{~kg} \mathrm{ha}^{-1}$, para uma aplicação de 134,6 kg ha-1 de $\mathrm{P}_{2} \mathrm{O}_{5}$ a lanço, e de $3.620 \mathrm{~kg} \mathrm{ha}^{-1}$, para uma aplicação de $155,0 \mathrm{~kg} \mathrm{ha}^{-1}$ de $\mathrm{P}_{2} \mathrm{O}_{5}$ no sulco de plantio.

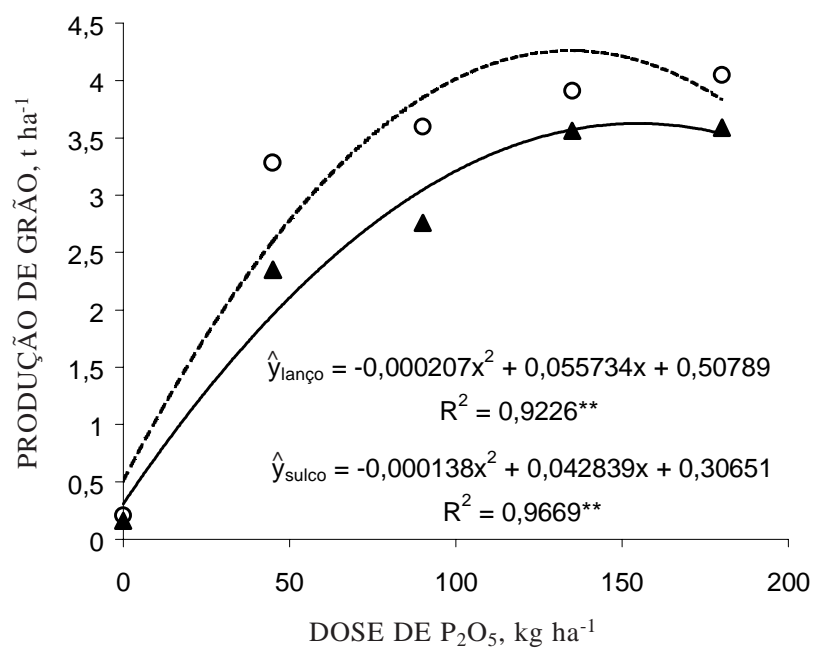

Figura 2. Produtividade de grãos de milho em resposta a doses de $\mathrm{P}_{2} \mathrm{O}_{5}$ adicionadas a lanço (--) e no sulco de plantio (-) em um solo de tabuleiro costeiro. 
Deacordo com estas observações, a incorporação do P a um volume maior de solo propiciou não apenas maior produção de grãos, mas também mostrou-se mais eficiente em termos de economia de adubo fosfatado (Figura 2). Tal diferença foi mais bem observada, quando se comparou a produtividade de grãos por $\mathrm{kg}$ de $\mathrm{P}_{2} \mathrm{O}_{5}$, no ponto de máxima produtividade dos dois modos de aplicação do adubo fosfatado, que foi de $31,6 \mathrm{~kg}$, para a adubação fosfatada a lanço, e de 23,4, para a real izada no sulco de plantio.

O fornecimento localizado de P no solo, de modo que apenas uma fração das raízes de milho entre em contato com este nutriente, resulta na compartimentalização do $P$ na parte aérea desta planta (Stryker et al., 1974). Esta compartimentalização é atribuída à organização vascular do milho e pode levar ao surgimento de desequilíbrios nutricionais em determinadas regiões da planta, quer pelo excesso, quer pela deficiência de $\mathrm{P}$ em relação a outros nutrientes, como o N (Novais et al., 1985; Alves et al., 1999), K, Ca e Mg (Alves et al., 1999).

Ainda que desequilíbrios como estes possam ter ocorrido nas plantas adubadas no sulco, estes não teriam sido suficientemente expressivos para alterar a eficiência de utilização do P foliar (EUP) por estas plantas, já que o modo de adubação não al terou esta variável no presente estudo (Quadro 1). A resposta das plantas de milho às doses de $\mathrm{P}_{2} \mathrm{O}_{5}$, em termos de EUP, foi quadrática com um máximo de $396 \mathrm{~g}$ de grãos por planta, para cada unidade percentual de

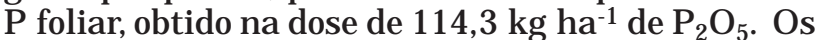
incrementos nos teores de $\mathrm{P}$ foliar proporcionados por doses de $\mathrm{P}_{2} \mathrm{O}_{5}$ superiores a esta não resultaram em aumento da EUP para a produtividade degrãos.
De acordo com estes resultados, conclui-se que a mai or produtividade de milho nas parcelas adubadas a lanço, comparativamenteà das adubadas em sulco, deve-se principalmente à maior absorção deste nutriente pelas plantas, enão a uma maior eficiência de utilização do $\mathrm{P}$ foliar pelas mesmas.

A adubação a lanço resultou ainda em maior produtividade deraízes na dose avaliada de $90 \mathrm{~kg} \mathrm{ha}^{-1}$ de $\mathrm{P}_{2} \mathrm{O}_{5}$ (Quadros 2 e 3). Além disso, observou-se uma tendência de maior distribuição dessas raízes em regiões de sol o mais distantes da planta (Quadro 3), comparativamente às plantas adubadas no sulco de plantio. Esta maior expansão radicular no solo adubado com $\mathrm{P}$ a lanço pode ter contribuído para aumentar a absorção de outros nutrientes e de água (Drake\& Stewart, 1950), o quetambém favoreceria o aumento da produtividade de grãos de milho.

Quadro 2. Análise de variância da produtividade de massa seca de raízes de milho, na dose de $90 \mathrm{~kg} \mathrm{ha}^{-1} \mathrm{P}_{2} \mathrm{O}_{5}$, considerando a forma de adição de $\mathbf{P}$ ao solo e as distâncias da planta

\begin{tabular}{lrc}
\hline & & Quadrado médio \\
\cline { 3 - 3 } & G.V. & Massa seca de raízes \\
\hline Bloco & 2 & $0,0450^{*}$ \\
Forma de adição do P (A) & 1 & $0,1104^{* *}$ \\
Distância da Planta (D) & 4 & $0,6903^{* *}$ \\
A x D & 4 & $0,0072^{\text {ns }}$ \\
Resíduo & 18 & 0,0094 \\
Total & 29 & \\
C.V. (\%) & & 21,76
\end{tabular}

**, * e ns: Significativos a 1 e 5\% e Não-significativo, respectivamente, pelo teste $\mathrm{F}$.

Quadro 3. Produção de massa seca de raízes nos modos de adição do adubo fosfatado em sulco e a lanço nos diversos intervalos de amostragem a partir do coleto, na dose de $90 \mathrm{~kg} \mathrm{ha}^{-1}$ de $\mathrm{P}_{2} \mathrm{O}_{5}$

\begin{tabular}{|c|c|c|c|c|c|}
\hline \multirow{3}{*}{$\begin{array}{c}\text { Distância de amostragem } \\
\mathrm{cm}\end{array}$} & \multicolumn{4}{|c|}{ Forma de adição do fósforo } & \multirow{3}{*}{$\begin{array}{l}\text { Média } \\
\mathrm{g} \mathrm{dm}^{-3}\end{array}$} \\
\hline & \multicolumn{2}{|c|}{ Sulco } & \multicolumn{2}{|c|}{ Lanço } & \\
\hline & $\mathrm{g} \mathrm{dm}^{-3}$ & $\%$ & $\mathrm{~g} \mathrm{dm}^{-3}$ & $\%$ & \\
\hline $\begin{array}{r}0-10 \\
10-20 \\
20-30 \\
30-40 \\
40-50\end{array}$ & $\begin{array}{l}1,02 \\
0,31 \\
0,20 \\
0,18 \\
0,20\end{array}$ & $\begin{array}{l}53^{(1)} \\
16 \\
10 \\
09 \\
10\end{array}$ & $\begin{array}{l}1,07 \\
0,37 \\
0,41 \\
0,31 \\
0,37\end{array}$ & $\begin{array}{l}42 \\
14 \\
16 \\
12 \\
14\end{array}$ & $\begin{array}{l}1,04 \mathrm{a}^{(2)} \\
0,34 \mathrm{~b} \\
0,30 \mathrm{~b} \\
0,24 \mathrm{~b} \\
0,28 \mathrm{~b}\end{array}$ \\
\hline Total & 1,91 & 100 & 2,53 & 100 & \\
\hline Média & $0,38 \mathrm{~B}^{(3)}$ & & $0,51 \mathrm{~A}$ & & \\
\hline
\end{tabular}

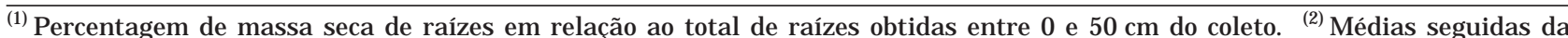
mesma letra não diferem entre si pelo teste de Tukey a 5\%. ${ }^{(3)}$ Comparação, pelo mesmo teste, entre formas de adição do fósforo. 


\section{CONCLUSÕES}

1. A adubação de $P$ a lanço, em solos da região estudada, resultou em mai or produtividade de milho, com maior economia de adubo fosfatado, comparativamente à adubação no sulco de plantio.

2. A maior absorção de $\mathrm{P}$ pelas plantas foi $\mathrm{a}$ principal causa do aumento de produtividade de milho nas parcel as adubadas a lanço, e não a maior eficiência de utilização do $P$ foliar por estas plantas.

\section{LITE RATURA CITADA}

ALVES, V.M.C.; MAGALHÃES, J.V.; VASCONCELLOS, C.A; NOVAIS, R.F.; BAHIA FILHO, A.F.C.; FRANÇA, G.E.; OLIVEIRA, C.A. \& FRANÇA, C.C.M. Acúmulo denitrogênio e de fósforo em plantas de milho afetadas pelo suprimento parcial de fósforo às raízes. R. Bras. Ci. Solo, 23:299-305, 1999.

ANGHINONI, I. \& BARBER, S.A. Predicting the most efficient phosphorus placement for corn. Soil Sci. Soc. Am. J., 44:1016-1020, 1980

ANGHINONI, I. Uso de fósforo pelo milho afetado pela fração de solo fertilizada com fosfato solúvel. R. Bras. Ci. Solo, 16:349-353, 1992.

BORKERT, C.M. \& BARBER, S.A. Soybean shoot and root growth and phosphorus concentration as affected by phosphorus placement. Soil Sci. Soc. Am. J., 49:152-155, 1985.

DRAKE, M. \& STEWART, E.H. Alfalfa fertility investigations in South Carolina. Soil Sci., 69:459-469, 1950.

EMPRESA BRASILEIRA DE PESQUISA AGROPECUÁRIA EMBRAPA. Centro Nacional de Pesquisa de Solos. Manual de métodos de análise de solo. Rio de J aneiro, 1997. 212p. (EMBRAPA-CNPS. Documentos; 1)

GOMEZ, K.A. \& GOMEZ, A.A. Statistical procedures for agricultural research with emphasis on rice. Los Baños, The International Rice Research Institute, 1976. 294p.
JACKSON, M.L. Soil chemical analysis. New J ersey, Prentice Hall, 1958. 498p.

KLEPKER, D. \& ANGHINONI, I. Phosphate uptake and corn root distribution as affected by fertilizer placement and soil tillage. Agron. Trends Agric. Sci., 1:111-115, 1993.

KLEPKER, D. \& ANGHINONI, I. Modos de adubação, absorção denutrientes erendimento de milho em diferentes preparos de solo. Pesq. Agropec. Gaúcha, 2:79-86, 1996.

MALAVOLTA, E.; VITTI, G.C. \& OLIVEIRA, S.A. Avaliação do estado nutricional das plantas. Piracicaba, Potafos, 1989. 201p.

MILLER, M.H.; BATES, T.E.; SIGH, D. \& BAWEJA, A.S. Response of corn to small amounts of fertilizer placed with the seed. I. Greenhouse studies. Agron. J ., 63:365-368, 1971.

NOVAIS, R.F. \& SMYTH, T.J. Fósforo em solo e planta em condições tropicais. Viçosa, U niversidadeFederal deViçosa, 1999. 399p.

NOVAIS, R.F.; FERREIRA, R.P.; NEVES, J.C.L. \& BARROS, N.F. Absorção de fósforo e crescimento do milho com sistema radicular parcialmente exposto a fonte de fósforo. Pesq. Agropec. Bras., 20:749-754, 1985.

PERYEA, F.J . Phosphate-fertilizer-induced salt toxicity of newly planted appletrees. Soil Sci. Soc. Am. ., 54:1778-1783, 1990.

SIDDIQI, M.Y. \& GLASS, D.M. Utilization index: a modified approach to the estimation and comparison of nutrient utilization efficiency in plants. J. Plant Nut., 4:289-302, 1981.

SILVA, D.J .; ALVARENGA, R.C.; ALVAREZ V., V.H. \& SOARES, P.C. Localização de fósforo e de cál cio no solo e seus efeitos sobre o desenvolvimento inicial do milho. R. Bras. Ci. Solo, 17:203-209, 1993.

SILVA, F.C.; EIRA, P.A.; RAIJ , B. van; SILVA, C.A.;ABREU, C.A.; GIANELLO, C.; PÉRES, D.V.; QUAGGIO, J.A.; TEDESCO, M.J .; ABREU, M.F. \& BARRETO,W.O. Análises químicas para avaliação da fertilidade do solo. In: SILVA, F.C., org. Manual de análises químicas de solos, plantas e fertilizantes. Brasília, Empresa Brasileira de Pesquisa Agropecuária. 1999. p.75-166.

STRYKER, R.B.; GI LLIAM,J.W. \& J ACKSON, W.A. Nonuniform transport of phosphorus from single roots to the leaves of Zea mays. Physiol. Plant., 30:231-239, 1974. 